Microbiol Spectr. 2016 December ; 4(6): . doi:10.1128/microbiolspec.UTI-0024-2016.

\title{
Innate Immune Responses to Bladder Infection
}

\author{
Byron W Hayes ${ }^{1}$ and Soman N Abraham ${ }^{1,2,3,4}$ \\ ${ }^{1}$ Department of Pathology, Duke University Medical Center, Durham, North Carolina 27710, USA \\ 2Department of Molecular Genetics \& Microbiology, Duke University Medical Center, Durham, \\ North Carolina 27710, USA \\ ${ }^{3}$ Department of Immunology, Duke University Medical Center, Durham, North Carolina 27710, \\ USA \\ ${ }^{4}$ Program in Emerging Infectious Diseases, Duke-National University of Singapore, Singapore
}

\begin{abstract}
Urinary tract infections (UTIs) are one of the most frequent bacterial infections of mankind. In spite of its frequency, the study of the immune system in the urinary tract has not attracted much attention. This could, in part, be attributable to the widespread use of antibiotics and similar antimicrobial agents which for many decades has been both highly effective and relatively inexpensive to administer. In light of the emergence of multidrug resistant bacteria among UTI isolates, interest in understanding the immune system in the urinary tract (UT) has grown. Several recent studies have revealed the existence of a powerful and highly coordinated innate immune system in the UT designed to rapidly clear infecting pathogens; however, it also evokes harmful side effects.
\end{abstract}

\section{INTRODUCTION}

The UT consists of the kidneys, ureters, bladder and urethra; all of which with the exception of the lower urethra are presumed to be sterile. Because of its close proximity to the gut, the lower UT is constantly exposed to a barrage of gut bacteria. However, the bladder remains for the most part free of infection. The resistance of the bladder to active microbial colonization is due to both anatomical design as well as secreted antimicrobial compounds of the urothelium. The apical face of the urothelium is covered by uroplakin plaques and is coated by mucus, which discourages adherence and invasion of most microorganisms $(1,2)$. Because of its role in storing urine for extended periods of time, the urothelium of the bladder has an additional role in protecting the underlying tissue from urine and its many toxic constituents. Since the bladder and urinary system as a whole need to constantly maintain the integrity of the urothelium, immune responses in the UT are often tightly regulated to minimize the extent of damage by quelling inflammation in a timely manner.

A rapid and vigorous response mediated by the immune system is largely responsible for guarding against bacterial infections that bypass the natural defenses of the urinary tract. In 
spite of this, some pathogens such as uropathogenic Escherichia coli (UPEC) are still able to colonize and infect this tract. UPEC is responsible for more than 80 percent of urinary tract infections (UTIs); the pathogen is believed to originate from the intestines as these bacteria can be regular components of the microbiome of the gastrointestinal tract (3). UPEC are especially adapted to infect the UT as they have the potential to multiply rapidly in urine and to bypass or overcome many of the natural barriers to infection. Beyond this, they appear capable of persisting for extended periods of time within the bladder epithelium often in a quiescent state, thereby contributing to chronic or recurrent UTI (4).

One in three women will have at least one UTI by age 24 and over half of all women will have at least one UTI in their lifetime (5). The cost of treatment in the United States alone is several billion dollars each year and growing (5). There is emerging concern of the involvement of multidrug resistant E. coli, many of which contribute to increased frequency of recurrence (6). In view of these concerns, there is a strong impetus to discover novel and alternative strategies to treat or manage UTIs. Valuable clues can be derived from understanding the natural immune processes and mechanisms involved in the host response to uropathogens in the UT. Our goal in this chapter is to briefly review key innate immune defense activities observed in the UT. Focus is placed on the bladder as most infections of the upper UT originate from bacteria ascending from the bladder (7). The adaptive immune responses will not be discussed here as they appear limited following bladder infections (8).

\section{CELLULAR COMPONENTS OF THE INNATE IMMUNE RESPONSE TO INFECTION}

\section{Epithelial Cells}

The first host cells invading uropathogens encounter upon entering the bladder are the superficial bladder epithelial cells (BECs) that form a tight, highly impregnable barrier. These cells are replete with receptors for various bacteria or their products. Evidence of pattern recognition receptor (PRR) activity can be seen soon after infection as a large burst in secretion of Interleukin-6 (IL-6) and IL-1 $\beta$ is detectable in the urine (9). These cytokines are typically the products of Toll-like receptor 4 (TLR4) and Nod-like receptor/Caspase 1 activity, respectively $(9,10)$. These cytokines along with other inflammatory mediators secreted by BECs result in a vigorous influx of immune cells to the epithelium to counter the bacterial challenge. BECs complement this cytokine response with secretion of several factors capable of directly killing bacteria. One such example is the cathelicidin LL-37 (11). mRNA expression for cathelicidin was observed to rise within 5 minutes of stimulation of epithelial cells with UPEC (11). In addition, knockout of the mouse LL-37 ortholog resulted in a sharp increase in UPEC load in the bladder of mice within one hour following infection, revealing the rapid and powerful antibacterial contribution of this antimicrobial peptide (AMP) (11). $\beta$-defensin is another AMP found in urine but this agent mainly originates from kidney epithelial cells rather than BECs (12). Both these AMPs also contribute to cytokine production and neutrophil recruitment in the bladder (13). Indeed, cathelicidin deficient mice had lower levels of 16 different inflammatory cytokines in bladder homogenates after infection, including IL- $1 \beta$ and IL-6. Ribonuclease 7 is another antimicrobial agent that has swift, broad spectrum microbicidal activity against many common uropathogens at basal 
levels (14). The soluble pattern recognition molecule pentraxin-related protein 3 (PTX3) was recently found to be relevant in defending against microbial infection of the UT (15). PTX3 is produced by many cell types including urothelial cells and its activity appears to be through stimulating complement mediated phagocytosis of pathogens. PTX3 levels after infection increased in the UT and knockout mice had a decreased ability to clear infection (15). Genetic mutations in the $P T X 3$ locus in humans also coincides with increased likelihood of UTIs (15).

In addition to forming a highly effective barrier to infection and urine and functioning as a source of soluble antimicrobial agents, BECs have a critical role in regulating the bladder volume to accommodate urine. BECs contain a large pool of RAB27 $\mathrm{b}^{+}$fusiform vesicles (16). When urine enters the bladder, these fusiform vesicles spontaneously exocytose into the plasma membrane in a cAMP dependent manner to provide the extra membranes required for bladder expansion. After voiding when the bladder volume needs to contract, the intracellular fusiform vesicles once again form by internalization of the RAB27b ${ }^{+}$ membrane $(16,17)$. UPEC have been found to coopt this unique property to gain entry into BECs. Upon binding to the apical surface of BECs, they are able to trigger a local burst of cAMP resulting in exocytosis of fusiform vesicles at the adherence site; when these $\mathrm{RAB} 27 \mathrm{~b}^{+}$vesicles are subsequently retracted from the cell surface, the bacteria are drawn into the cell where they reside encased in RAB27b ${ }^{+}$vesicles (17). Remarkably, BECs have an autonomous defense system capable of sensing intruding bacteria and initiating mechanisms to eject bacteria from their intracellular refuge. This activity is initiated within minutes of bacterial entry and is triggered by TLR4 localized in the vesicles encasing bacteria (18). This immune sensory molecule mobilizes the cellular trafficking machinery typically employed to export hormones to instead export UPEC from infected BECs. TLR4 signaling was found to trigger K33-linked polyubiquitination of the signaling substrate, TRAF3, which was then detected by RalGDS, a guanine nucleotide exchange factor (GEF) that precipitated the assembly of the exocyst complex, a powerful exporter of subcellular vesicles (19). Interestingly, not all intracellular bacteria in BECs are expelled from $\mathrm{RAB} 27 \mathrm{~b}^{+}$vesicles in this manner. It was observed that an appreciable number of UPEC are capable of escaping intracellular vesicles in BECs and entering the cytosol, at which point a second export mechanism appears to be activated. This pathway is initiated by the cell's autophagy system which encapsulates the bacterium in a autophagosome and transports it to the lysosome via the multivesicular body. However, UPEC have the capacity to neutralize the lysosome in order to survive this environment (20). BECs seem to counter this pathogen response by triggering expulsion of UPEC from malfunctioning lysosomes employing transient receptor potential (TRP) mucolipin 3 (TRPML3), a cation channel on the lysosome which becomes activated when the $\mathrm{pH}$ within the lysosome increases. UPEC expelled from lysosomes were found to be encased in a host cell membrane (20). While the expulsion of UPEC from RAB27b ${ }^{+}$vesicles occurs soon after bacterial entry, exocytosis from lysosomes is only initiated 4 hours or more after infection. Together these export mechanisms are highly effective, resulting in the expulsion by 24 hours of over $80 \%$ of bacteria that had gained entry into BECs (17).

Another powerful property possessed by BECs to combat infections is undergoing cell death and shedding into the urine, thus eliminating all of the adherent and intracellular bacteria 
associated with these cells. Urine of heavily infected subjects are rich in exfoliated BECs indicating that exfoliation of BECs is a frequently employed host defense mechanism (21, 22). This capacity of infected BECs to trigger cell death is a double-edged sword as it allows for elimination of large congregates of bacteria associated with BECs but also exposes underlying cells to both toxic urine as well as any bacteria still present in the urine (23). To counter this drastic action, the basal epithelium promptly shifts to a rapid proliferative state to quickly replace the shed cells within hours and restore the urothelial barrier $(24,25)$.

\section{Neutrophil, Mast cells and Macrophages}

Arguably the first recruited immune cells to target infecting bacteria in the bladder are neutrophils. These innate immune cells are the primary cells responsible for bacterial elimination once the inflammatory response is triggered. When their PRRs are stimulated upon entry of bacteria, BECs produce IL-8 which leads to the recruitment of neutrophils to the superficial epithelium and the bladder lumen, where infecting bacteria are found (26). Recruited neutrophils exit the blood vessels and migrate through the lamina propria to penetrate the basement membrane. Although neutrophil migration to the epithelium is primarily in response to IL-8, its ability to also express metalloproteinase- 9 appears to be key to its ability to penetrate the basement membrane (27). Once the neutrophils cross the basement membrane they readily circumvent multiple layers of intermediate epithelial cells before reaching the superficial epithelium which is the final epithelial cell layer before the neutrophils enter the bladder lumen. Since large numbers of neutrophils are found in the urine, it is clear that these neutrophils are capable of breaking through the tight junctions between adjoining superficial epithelial cells. Neutrophil recruitment into the UT is typically swift and often proportional to bacterial load (28). Neutrophil counts in the epithelial region following bladder infection reveal that these phagocytic cells arrive within 2 hours of infection but appear to peak by 6 hours (28). Their numbers correspondingly decrease as bacteria are cleared. A powerful indicator of the importance of neutrophils to bacterial clearance comes from studies in mouse strains with neutrophil response defects. These mice exhibited significantly increased susceptibility to UTIs and decreased ability to clear bacterial infections (29).

Much of the antibacterial actions of neutrophils in the UT can be ascribed to the capacity of these cells to engulf and intracellularly degrade bacteria. Promoting this activity is the neutrophil's capacity to secrete prestored PTX3, which serves as an opsonin, to coat bacteria and facilitate their uptake. Neutrophils from PTX3 knockout mice are known to display decreased phagocytic activity that can be restored by opsonizing bacteria with soluble PTX3 (15). Although neutrophils are critical for bacterial clearance, they are also largely responsible for much of the cytotoxicity associated with bacterial infections of the UT (30). Neutrophil mediated tissue damage is ascribable to reactive oxygen species and other cytotoxic products that these cells release upon reaching the urinary epithelium (30). Recently, treatment with nonsteroidal anti-inflammatory drugs (NSAIDS) or COX-2 inhibitors was found to exhibit a remarkable therapeutic effect in experimental mouse models (30). Use of inhibitors of COX-2 in infected mice revealed the COX-2 inhibitors exhibit a dramatic ability to limit neutrophil migration into the UT. Limited neutrophil influx in the UT was closely associated with markedly less pyuria and mucosal damage. The 
COX-2 inhibitors did not impact the exfoliation of infected BECs and other natural clearance activities in the UT. Interestingly, inhibiting COX-2 in a mouse recurrent UTI model was found to reduce the likelihood of chronic cystitis (30) as the neutrophil associated tissue damage was a major contributor to chronic inflammation. In addition, serum from women who developed a recurrent infection had elevated levels of cytokines associated with neutrophil development and chemotaxis (30). In view of the severe pathology evoked by recruited neutrophils during UTIs, it is conceivable that the large presence of neutrophils in the urine is a byproduct of host defenses aimed at eliminating these cytotoxic cells from the surrounding tissue. It is unclear how effective neutrophils are in killing bacteria in urine, but the presence of large numbers of neutrophils in the urine will ensure that they are immediately eliminated during voiding (29).

One of the most prominent resident innate immune cell types in the UT are mast cells. These cells are found in much higher numbers in the bladder compared to the kidneys, perhaps implying a more prominent role in the former organ. In the bladder, mast cells are primarily localized to the lamina propria and in the detrusor muscles, although they appear capable of relocating and increasing in number following infection. Mast cells are sentinel cells that initiate the early immune responses to microbial or other insults. Prestored in their granules are a large pool of proinflammatory mediators that can be immediately released upon mast cell activation. The speed and magnitude of the ensuing inflammatory response is largely dependent on the contents of these mast cell granules. Within the first hour of infection, histamine and other prestored proinflammatory mediators typically found in granules can be found in urine $(31,32)$. Prestored mast cell products such as TNF contribute to the early neutrophil response in the bladder as mast cell deficient mice evoke a limited neutrophil response to bacterial infection compared to mast cell sufficient mice (32). Not surprisingly, bacterial clearance in the bladder and kidneys of these mast cell deficient mice are also limited (33). Although mast cell membranes are replete with PRRs they also express membrane receptors such as CD48, a GPI anchored molecule that serves as a receptor for the bacterial adhesin FimH, expressed by type I fimbriated E. coli (34). Since mast cells lie underneath the epithelium it is unlikely that these cells make contact with bacteria or their products early in the infection. It is more likely that mast cells are activated by "danger signals" secreted by stressed or damaged epithelial cells such as ATP, IL-33 and $\beta$-defensin, all of which are potent mast cell activators (35-37). In addition to their role in initiating inflammation during infection, mast cells also appear to be important in establishing homeostasis and accelerating tissue recovery after the infection subsides. Recently, it was reported that several hours after bladder infection, local mast cells were abruptly found to express the immunosuppressive cytokine, IL-10 (33). The timing of IL-10 release appeared to coincide with epithelial shedding and when recovery of the epithelium needed to occur. Because the constituents of urine can be highly toxic to the underlying tissue, it is imperative that the bladder epithelium be reconstituted as quickly as possible. Since tissue regeneration cannot occur during active inflammation, inflammation in the bladder epithelium needs to be sharply curtailed, hence the requirement for mast cell derived IL-10. Interestingly, an apparent side effect of this mast cell derived IL-10 appears to be premature abrogation of the adaptive immune response. Studies in mice revealed that mast cell derived IL-10 negatively impacted functional activity of both dendritic cells and $\mathrm{T}$ cells in the iliac lymph nodes that 
drain the bladder (33). Additionally, mast cell derived IL-10 could potentially contribute to premature resolution of inflammation in the bladder without complete eradication of infecting bacteria in the bladder. A common observation in mouse models of bladder infection is that a significant population of residual bacteria remain in the bladder long after apparent resolution of the infection $(33,38,39)$.

Resident tissue and recruited macrophages also appear to play a critical role in the highly orchestrated neutrophil response to bladder infections. Macrophages that are resident in the lamina propria of the bladder tend to be $\mathrm{Ly}^{6} \mathrm{C}^{-}$macrophages, which upon infection recruit both neutrophils and $\mathrm{Ly}_{6} \mathrm{C}^{+}$macrophages out of the circulation into the bladder lamina propria through the secretion of chemokines such as CXCL1 and CXCL2 (27). Interestingly, there appears to be a checkpoint in the basement membrane which prevents newly recruited neutrophils from reaching the epithelium. It now appears that specific communication between resident $\mathrm{Ly} 6 \mathrm{C}^{-}$macrophages and newly recruited $\mathrm{Ly}_{6 \mathrm{C}} \mathrm{C}^{+}$macrophage subtypes is necessary before neutrophils can break through the collagen IV barrier that constitutes the basement membrane. Newly recruited macrophages secrete TNF which causes the resident macrophage subset to secrete CXCL2, a powerful chemokine which in turn induces the neutrophil pool in the lamina propria to produce matrix metalloproteinase-9, which is necessary for neutrophil breakthrough across the basement membrane barrier to reach the epithelium (27). Thus, it would appear that bladder macrophages critical regulatory control of the functional activity of recruited neutrophils. Concomitant with their role in promoting neutrophil influx and migration into the bladder epithelium, macrophages are known to temper neutrophil responses by ingesting and removing apoptotic neutrophils to promote early resolution of inflammation (40). Like mast cells, resident macrophages of the bladder appear to impede development of adaptive immune responses following infection. These resident macrophages appear to successfully compete with dendritic cells in bacterial uptake but unlike the latter cells, they are not efficient at antigen presentation resulting in a poor adaptive immune response (41).

Studies in mice have revealed that other immune cells in the bladder that contribute to the innate immune response to bacterial infection include natural killer (NK) and $\gamma \delta \mathrm{T}$ cells (42-44). How these bladder resident immune cells directly combat bacteria and also collaborate with other immune cells is currently being investigated.

\section{IMMUNE BREAKDOWN AND UTI RISK FACTORS}

UTIs remain frequent and even appear to be on the rise, especially within the rapidly growing aging population. Although UTIs are strongly associated with UPEC expressing a collection of virulence factors including type 1 fimbriae, flagella and hemolysin which together enable the bacteria to successfully colonize the unique microenvironment of the bladder and kidney (7), many of these infections can still be traced back to specific defects in one or more components of the innate immune system.

Often, there is a genetic basis for the breakdown in innate immune defenses. For example, polymorphisms in TLR1, TLR4, CXCR1, among others appear to predispose to recurrence of UTIs in patients (45). The importance of these genes in the early recognition of infecting 
bacteria and/or in recruiting appropriate immune cells to infection sites can be readily gleaned from experimental infections in mice deficient in these genes (45). While not strictly considered a breakdown of immune defenses, the expression of certain blood group antigens on cells lining the UT can predispose to UTIs. These antigens serve as receptors for specific fimbriae typically expressed by UPEC (46). Consequently, their presence on uroepithelial cell surfaces can promote bacterial adherence and promote infection at these sites. In view of their impact, some have proposed that $\mathrm{P}$ blood group status may be used as a risk marker for pyelonephritis (45).

As people age, the efficiency of their immune system in combating microbial challenge decreases due to immune senescence (47). For example, neutrophil migration and their associated microbicidal activity are severely impaired in aged individuals (48); consequently, these individuals exhibit a limited capacity to effectively clear bacteria once infected. In aging women, in addition to defective immune cells, decreasing levels of estrogen appear to be contributing factor as postmenopausal women are more susceptible to UTIs than their premenopausal counterparts $(49,50)$. Estrogen receptors are present on epithelial cells lining the lower UT. When mice subjected to ovariectomy, to simulate menopause, were examined, they were found to exhibit higher bacterial loads in the bladder and an overall delayed clearance of UPEC compared to controls (51). These mice also experienced more severe inflammation and decreased exfoliation of their uroepithelial cells, a major contributing factor to bacterial load in the UT. Although estrogen supplementation does not reduce the level of bacteria in the urine, in a mouse model, estrogen supplementation decreased the levels of proinflammatory cytokines and decrease the extent of damage in the bladder during the natural immune response (51).

While specific defects in various components of the immune system predisposes for the development of UTIs, some highly host-adapted bacterial strains appear capable of establishing infection by actively suppressing most of the local immune responses in the UT. In these cases, not only is there no accompanying inflammatory response but there is very little infection-associated pathology in the UT. Consequently, the infecting bacteria appear to grow unrestricted in the urine contained in the bladder lumen of the infected individual. Because the patients experience no discomforting symptoms, the infection is referred to as asymptomatic bacteriuria. Recent studies employing the asymptomatic E. coli 83972 strain have revealed the underlying basis for bacterial suppression of pathology in the UT. One important trait possessed by asymptomatic bacteria is that they fail to express most of the virulence factors traditionally associated with UPEC (52). This property allows the bacteria to avoid activating immune surveillance molecules found in cells that line the UT. An even more potent trait found in asymptomatic bacteria is that of suppressing RNA polymerase II (Pol II)-dependent gene expression in host cells (53). This bacteria-induced effect on Pol IIdependent transcription factors was observed in both bladder as well as renal cells in vitro. In patients infected with E. coli 83972, the expression of $60 \%$ of all regulated genes was markedly reduced after 24 hours (53). Many of these regulated genes are found in diseaseassociated innate immune response pathways, defined by TLR4, IRF-3 and IRF-7 and include immune surveillance molecules NOD1 and TLR5 (45). This gene suppression was long lasting as it was evident in patients a week after inoculation of asymptomatic UPEC (53). Since asymptomatic E. coli such as the 83972 strain are better suited at long term 
survival in the UT than their uropathogenic counterparts that express many of the traditional virulence factors, it has been proposed that these bacteria be used as probiotics to displace more harmful strains or even prevent their infection of the bladder $(53,54)$. Since the bladder is mostly sterile, asymptomatic strains would have very little competition establishing themselves in the bladder of subjects prone to recurrent UTIs and theoretically these bacteria could discourage the establishment of more virulent strains. However, a recent clinical trial using E. coli 83972 for prevention of catheter associated urinary tract infections did not corroborate this theory; patients in which the asymptomatic strain successfully colonized still had uropathogens present and more than half of these patients developed a UTI (55). Intriguingly, the individuals that developed a UTI had a lower assortment of bacteria in their urine, suggesting microbial diversity may play a protective role in the UT (55).

\section{CONCLUSION}

Recent studies revealed that a coordinated multicomponent immune defense program is activated by resident BECs, mast cells and macrophages when bacteria reach the bladder. In addition to directly attacking the invading pathogen, their individual responses are largely aimed at rapidly recruiting neutrophils into the bladder to clear the pathogens. Large populations of neutrophils recruited into the bladder are highly efficacious in eliminating infecting bacteria from the UT. However, these recruited neutrophils are also extremely cytotoxic to the surrounding tissue. Indeed, much of the tissue damage incurred during UTIs is attributable to recruited neutrophils. Not surprisingly, each of the bladder resident cell types have distinct mechanisms to reduce neutrophil numbers or activity at inflamed sites. Epithelial cells reduce neutrophil numbers by recruiting them into the urine where they are eliminated during voiding. Mast cells impede neutrophils through secretion of IL-10 when epithelial cells begin to shed and compromise their barrier function. Macrophages eliminate spent neutrophils by phagocytosis and subsets of these cells also appear capable of restricting inappropriate neutrophil recruitment into the epithelium by regulating the permeability of the basement membrane. Studies in mice and genetic analysis of patients prone to recurrent UTIs reveal that defects in the one or more of these immune responses predispose to the severity and persistence of UTIs.

Cumulatively, these observations represent only a glimpse of the complexity of the immune responses evoked in the bladder. More extensive studies in animal models coupled with immune monitoring of UTI prone populations during acute and chronic infections are required before meaningful therapeutic strategies can be developed.

\section{Acknowledgments}

This work was funded by U.S. National Institutes of Health grants: U01-AI082107; R01-AI096305; R56DK095198.

\section{References}

1. Wu XR, Kong XP, Pellicer A, Kreibich G, Sun TT. Uroplakins in urothelial biology, function, and disease. Kidney Int. 2009; 75:1153-1165. [PubMed: 19340092]

Microbiol Spectr. Author manuscript; available in PMC 2017 December 01. 
2. Grist M, Chakraborty J. Identification of a mucin layer in the urinary bladder. Urology. 1994; 44:2633. [PubMed: 7518980]

3. Ronald A. The etiology of urinary tract infection: traditional and emerging pathogens. Dis Mon. 2003; 49:71-82. [PubMed: 12601338]

4. Rosen DA, Hooton TM, Stamm WE, Humphrey PA, Hultgren SJ. Detection of intracellular bacterial communities in human urinary tract infection. PLoS Med. 2007; 4:e329. [PubMed: 18092884]

5. Foxman B. Epidemiology of urinary tract infections: incidence, morbidity, and economic costs. Dis Mon. 2003; 49:53-70. [PubMed: 12601337]

6. Foxman B. The epidemiology of urinary tract infection. Nat Rev Urol. 2010; 7:653-660. [PubMed: 21139641]

7. Nielubowicz GR, Mobley HL. Host-pathogen interactions in urinary tract infection. Nat Rev Urol. 2010; 7:430-441. [PubMed: 20647992]

8. Ratner JJ, Thomas VL, Sanford BA, Forland M. Bacteria-specific antibody in the urine of patients with acute pyelonephritis and cystitis. J Infect Dis. 1981; 143:404-412. [PubMed: 7014730]

9. Song J, Duncan MJ, Li G, Chan C, Grady R, Stapleton A, Abraham SN. A novel TLR4-mediated signaling pathway leading to IL-6 responses in human bladder epithelial cells. PLoS Pathog. 2007; 3:e60. [PubMed: 17465679]

10. Nagamatsu K, Hannan TJ, Guest RL, Kostakioti M, Hadjifrangiskou M, Binkley J, Dodson K, Raivio TL, Hultgren SJ. Dysregulation of Escherichia coli a-hemolysin expression alters the course of acute and persistent urinary tract infection. Proc Natl Acad Sci USA. 2015; 112:E871E880. [PubMed: 25675528]

11. Chromek M, Slamová Z, Bergman P, Kovács L, Podracká L, Ehrén I, Hökfelt T, Gudmundsson GH, Gallo RL, Agerberth B, Brauner A. The antimicrobial peptide cathelicidin protects the urinary tract against invasive bacterial infection. Nat Med. 2006; 12:636-641. [PubMed: 16751768]

12. Valore EV, Park CH, Quayle AJ, Wiles KR, McCray PB Jr, Ganz T. Human beta-defensin-1: an antimicrobial peptide of urogenital tissues. J Clin Invest. 1998; 101:1633-1642. [PubMed: 9541493]

13. Danka ES, Hunstad DA. Cathelicidin augments epithelial receptivity and pathogenesis in experimental Escherichia coli cystitis. J Infect Dis. 2015; 211:1164-1173. [PubMed: 25336727]

14. Spencer JD, Schwaderer AL, Wang H, Bartz J, Kline J, Eichler T, DeSouza KR, Sims-Lucas S, Baker P, Hains DS. Ribonuclease 7, an antimicrobial peptide upregulated during infection, contributes to microbial defense of the human urinary tract. Kidney Int. 2013; 83:615-625. [PubMed: 23302724]

15. Jaillon S, Moalli F, Ragnarsdottir B, Bonavita E, Puthia M, Riva F, Barbati E, Nebuloni M, Cvetko Krajinovic L, Markotic A, Valentino S, Doni A, Tartari S, Graziani G, Montanelli A, Delneste Y, Svanborg C, Garlanda C, Mantovani A. The humoral pattern recognition molecule PTX3 is a key component of innate immunity against urinary tract infection. Immunity. 2014; 40:621-632. [PubMed: 24745336]

16. Chen Y, Guo X, Deng FM, Liang FX, Sun W, Ren M, Izumi T, Sabatini DD, Sun TT, Kreibich G. $\mathrm{Rab} 27 \mathrm{~b}$ is associated with fusiform vesicles and may be involved in targeting uroplakins to urothelial apical membranes. Proc Natl Acad Sci USA. 2003; 100:14012-14017. [PubMed: 14625374]

17. Bishop BL, Duncan MJ, Song J, Li G, Zaas D, Abraham SN. Cyclic AMP-regulated exocytosis of Escherichia coli from infected bladder epithelial cells. Nat Med. 2007; 13:625-630. [PubMed: 17417648]

18. Song J, Bishop BL, Li G, Grady R, Stapleton A, Abraham SN. TLR4-mediated expulsion of bacteria from infected bladder epithelial cells. Proc Natl Acad Sci USA. 2009; 106:14966-14971. [PubMed: 19706440]

19. Miao Y, Wu J, Abraham SN. Ubiquitination of Innate Immune Regulator TRAF3 Orchestrates Expulsion of Intracellular Bacteria by Exocyst Complex. Immunity. 2016; 45:94-105. [PubMed: 27438768]

20. Miao Y, Li G, Zhang X, Xu H, Abraham SN. A TRP Channel Senses Lysosome Neutralization by Pathogens to Trigger Their Expulsion. Cell. 2015; 161:1306-1319. [PubMed: 26027738] 
21. Elliott TSJ, Reed L, Slack RCB, Bishop MC. Bacteriology and ultrastructure of the bladder in patients with urinary tract infections. J Infect. 1985; 11:191-199. [PubMed: 3936879]

22. Cheng Y, Chen Z, Gawthorne JA, Mukerjee C, Varettas K, Mansfield KJ, Schembri MA, Moore KH. Detection of intracellular bacteria in exfoliated urothelial cells from women with urge incontinence. Pathog Dis. 2016; 74:74.

23. Mulvey MA, Lopez-Boado YS, Wilson CL, Roth R, Parks WC, Heuser J, Hultgren SJ. Induction and evasion of host defenses by type 1-piliated uropathogenic Escherichia coli. Science. 1998; 282:1494-1497. [PubMed: 9822381]

24. Mysorekar IU, Isaacson-Schmid M, Walker JN, Mills JC, Hultgren SJ. Bone morphogenetic protein 4 signaling regulates epithelial renewal in the urinary tract in response to uropathogenic infection. Cell Host Microbe. 2009; 5:463-475. [PubMed: 19454350]

25. Shin K, Lee J, Guo N, Kim J, Lim A, Qu L, Mysorekar IU, Beachy PA. Hedgehog/Wnt feedback supports regenerative proliferation of epithelial stem cells in bladder. Nature. 2011; 472:110-114. [PubMed: 21389986]

26. Godaly G, Bergsten G, Hang L, Fischer H, Frendéus B, Lundstedt AC, Samuelsson M, Samuelsson $\mathrm{P}$, Svanborg C. Neutrophil recruitment, chemokine receptors, and resistance to mucosal infection. J Leukoc Biol. 2001; 69:899-906. [PubMed: 11404374]

27. Schiwon M, Weisheit C, Franken L, Gutweiler S, Dixit A, Meyer-Schwesinger C, Pohl JM, Maurice NJ, Thiebes S, Lorenz K, Quast T, Fuhrmann M, Baumgarten G, Lohse MJ, Opdenakker G, Bernhagen J, Bucala R, Panzer U, Kolanus W, Gröne HJ, Garbi N, Kastenmüller W, Knolle PA, Kurts C, Engel DR. Crosstalk between sentinel and helper macrophages permits neutrophil migration into infected uroepithelium. Cell. 2014; 156:456-468. [PubMed: 24485454]

28. Shahin RD, Engberg I, Hagberg L, Svanborg Edén C. Neutrophil recruitment and bacterial clearance correlated with LPS responsiveness in local gram-negative infection. J Immunol. 1987; 138:3475-3480. [PubMed: 3553327]

29. Haraoka M, Hang L, Frendéus B, Godaly G, Burdick M, Strieter R, Svanborg C. Neutrophil recruitment and resistance to urinary tract infection. J Infect Dis. 1999; 180:1220-1229. [PubMed: 10479151]

30. Hannan TJ, Roberts PL, Riehl TE, van der Post S, Binkley JM, Schwartz DJ, Miyoshi H, Mack M, Schwendener RA, Hooton TM, Stappenbeck TS, Hansson GC, Stenson WF, Colonna M, Stapleton AE, Hultgren SJ. Inhibition of Cyclooxygenase-2 Prevents Chronic and Recurrent Cystitis. EBioMedicine. 2014; 1:46-57. [PubMed: 26125048]

31. Abraham SN, St John AL. Mast cell-orchestrated immunity to pathogens. Nat Rev Immunol. 2010; 10:440-452. [PubMed: 20498670]

32. Abraham S, Shin J, Malaviya R. Type 1 fimbriated Escherichia coli-mast cell interactions in cystitis. J Infect Dis. 2001; 183(Suppl 1):S51-S55. [PubMed: 11171015]

33. Chan CY, St John AL, Abraham SN. Mast cell interleukin-10 drives localized tolerance in chronic bladder infection. Immunity. 2013; 38:349-359. [PubMed: 23415912]

34. Malaviya R, Gao Z, Thankavel K, van der Merwe PA, Abraham SN. The mast cell tumor necrosis factor a response to FimH-expressing Escherichia coli is mediated by the glycosylphosphatidylinositol-anchored molecule CD48. Proc Natl Acad Sci USA. 1999; 96:8110 8115. [PubMed: 10393956]

35. Soruri A, Grigat J, Forssmann U, Riggert J, Zwirner J. beta-Defensins chemoattract macrophages and mast cells but not lymphocytes and dendritic cells: CCR6 is not involved. Eur J Immunol. 2007; 37:2474-2486. [PubMed: 17705135]

36. Säve S, Persson K. Extracellular ATP and P2Y receptor activation induce a proinflammatory host response in the human urinary tract. Infect Immun. 2010; 78:3609-3615. [PubMed: 20515921]

37. Jang TY, Kim YH. Interleukin-33 and Mast Cells Bridge Innate and Adaptive Immunity: From the Allergologist's Perspective. Int Neurourol J. 2015; 19:142-150. [PubMed: 26620895]

38. Mysorekar IU, Hultgren SJ. Mechanisms of uropathogenic Escherichia coli persistence and eradication from the urinary tract. Proc Natl Acad Sci USA. 2006; 103:14170-14175. [PubMed: 16968784] 
39. Mulvey MA, Schilling JD, Hultgren SJ. Establishment of a persistent Escherichia coli reservoir during the acute phase of a bladder infection. Infect Immun. 2001; 69:4572-4579. [PubMed: 11402001]

40. Michlewska S, Dransfield I, Megson IL, Rossi AG. Macrophage phagocytosis of apoptotic neutrophils is critically regulated by the opposing actions of pro-inflammatory and antiinflammatory agents: key role for TNF-alpha. FASEB J. 2009; 23:844-854. [PubMed: 18971259]

41. Mora-Bau G, Platt AM, van Rooijen N, Randolph GJ, Albert ML, Ingersoll MA. Macrophages Subvert Adaptive Immunity to Urinary Tract Infection. PLoS Pathog. 2015; 11:e1005044. [PubMed: 26182347]

42. Jones-Carson J, Balish E, Uehling DT. Susceptibility of immunodeficient gene-knockout mice to urinary tract infection. J Urol. 1999; 161:338-341. [PubMed: 10037434]

43. Sivick KE, Schaller MA, Smith SN, Mobley HL. The innate immune response to uropathogenic Escherichia coli involves IL-17A in a murine model of urinary tract infection. J Immunol. 2010; 184:2065-2075. [PubMed: 20083670]

44. Engel D, Dobrindt U, Tittel A, Peters P, Maurer J, Gütgemann I, Kaissling B, Kuziel W, Jung S, Kurts C. Tumor necrosis factor alpha- and inducible nitric oxide synthase-producing dendritic cells are rapidly recruited to the bladder in urinary tract infection but are dispensable for bacterial clearance. Infect Immun. 2006; 74:6100-6107. [PubMed: 16966414]

45. Godaly G, Ambite I, Svanborg C. Innate immunity and genetic determinants of urinary tract infection susceptibility. Curr Opin Infect Dis. 2015; 28:88-96. [PubMed: 25539411]

46. Lichodziejewska-Niemierko M, Topley N, Smith C, Verrier-Jones K, Williams JD. P1 blood group phenotype, secretor status in patients with urinary tract infections. Clin Nephrol. 1995; 44:376379. [PubMed: 8719549]

47. Ginaldi L, Loreto MF, Corsi MP, Modesti M, De Martinis M. Immunosenescence and infectious diseases. Microbes Infect. 2001; 3:851-857. [PubMed: 11580980]

48. Ginaldi L, De Martinis M, D’Ostilio A, Marini L, Loreto MF, Quaglino D. The immune system in the elderly: III. Innate immunity. Immunol Res. 1999; 20:117-126. [PubMed: 10580637]

49. Hextall A, Cardozo L. The role of estrogen supplementation in lower urinary tract dysfunction. Int Urogynecol J Pelvic Floor Dysfunct. 2001; 12:258-261. [PubMed: 11569655]

50. Foxman B. Urinary Tract Infection in Postmenopausal Women. Curr Infect Dis Rep. 1999; 1:367370. [PubMed: 11095810]

51. Wang C, Symington JW, Ma E, Cao B, Mysorekar IU. Estrogenic modulation of uropathogenic Escherichia coli infection pathogenesis in a murine menopause model. Infect Immun. 2013; 81:733-739. [PubMed: 23264047]

52. Klemm P, Roos V, Ulett GC, Svanborg C, Schembri MA. Molecular characterization of the Escherichia coli asymptomatic bacteriuria strain 83972: the taming of a pathogen. Infect Immun. 2006; 74:781-785. [PubMed: 16369040]

53. Lutay N, Ambite I, Grönberg Hernandez J, Rydström G, Ragnarsdóttir B, Puthia M, Nadeem A, Zhang J, Storm P, Dobrindt U, Wullt B, Svanborg C. Bacterial control of host gene expression through RNA polymerase II. J Clin Invest. 2013; 123:2366-2379. [PubMed: 23728172]

54. Ferrières L, Hancock V, Klemm P. Biofilm exclusion of uropathogenic bacteria by selected asymptomatic bacteriuria Escherichia coli strains. Microbiology. 2007; 153:1711-1719. [PubMed: 17526829]

55. Horwitz D, McCue T, Mapes AC, Ajami NJ, Petrosino JF, Ramig RF, Trautner BW. Decreased microbiota diversity associated with urinary tract infection in a trial of bacterial interference. $\mathrm{J}$ Infect. 2015; 71:358-367. [PubMed: 26048203] 